\title{
Validación de "The Parental Stressor Scale Infant Hospitalization modificado, al español" en una unidad cuidados intensivos pediátricos
}

\author{
Validation of "The Parental Stressor Scale Infant Hospitalization modified, \\ to Spanish" in a pediatric intensive care unit
}

\author{
Sandra Navarro-Tapia ${ }^{\mathrm{a}}$, Muriel Ramírez $^{\mathrm{b}}$, Cristián Clavería $^{\mathrm{c}}$, Yerko Molina ${ }^{\mathrm{d}, \mathrm{e}}$
}

aEnfermera-Matrona, Mg. Psicologia Social, Especialista Recien Nacido Alto Riesgo. Profesor Asociado Adjunto Escuela de Enfermería Facultad de Medicina, Pontificia Universidad Católica de Chile

bEnfermera. Unidad de Cuidados Intensivos Pediátricos, Hospital Clínico de la Red de Salud UC CHRISTUS

'Cardiologo e Intensivista Pediátrico, División de Pediatría. Facultad de Medicina, Pontificia Universidad Católica de Chile

dPsicólogo, Mg Psicología de la Salud. Escuela de Enfermería Facultad de Medicina, de la Pontificia Universidad Católica de Chile

eFacultad de Psicología de la Universidad Adolfo Ibáñez

Recibido: 20 de diciembre de 2018; Aceptado: 21 de marzo de 2019

\begin{abstract}
Resumen
Introducción: Las unidades de cuidados intensivos son conocidas como ambientes de alto estrés para los familiares, este puede ser mayor cuando el paciente es un hijo/hija y los padres deben enfrentar la enfermedad y el ambiente hospitalario. El instrumento "The Parental Stressor Scale Infant Hospitalization (PSSIH), es una herramienta utilizada para medir estresores en Unidades de Cuidados Intensivos Pediátricos (UCIP), sin embargo, la escala no está validada en Chile. El objetivo de este estudio es validar y adaptar culturalmente el instrumento "The Parental Stressor Scale Infant Hospitalization modificado" en madres/padres de niños hospitalizados en la UCIP de un Hospital Universitario. Método: Estudio instrumental de validación. Luego de traducir y contratraducir la versión en inglés del instrumento, un grupo de 10 profesionales expertos evaluó la adaptación al español. Luego 10 padres/ madres de niños hospitalizados en la UCIP evaluaron la comprensión del instrumento. Las propiedades psicométricas del instrumento fueron evaluadas utilizando un análisis factorial exploratorio y alfa de Cronbach. Resultados: Se aplicó en una muestra de 221 padres/madres la versión chilena del "PSSIH modificado", tuvo mínimas modificaciones semánticas y los jueces expertos consideraron adecuado el instrumento, por lo que no fue necesario borrar ningún ítem. Se eligió la solución de 3 dimensiones, que explicaron el $48,89 \%$ de la varianza total del instrumento. El alfa de Cronbach fue de $0,885,0,902$ y 0,703 respectivamente para cada dimensión. Conclusión: El PSSIH modificado ha demostrado ser un instrumento confiable y valido en una muestra de niños chilenos hospitalizados en una Unidad de Cuidados Intensivos Pediátricos de un hospital universitario , cuyo nombre en español es "Escala de Evaluación de Estresores Parentales en niños hospitalizados en Unidades de Cuidados Intensivos Pediátricos” (EEEP - UCIP).
\end{abstract}

Palabras clave: Estrés parental; hospitalización; cuidados intensivos pediátricos; estresores parentales

Correspondencia:

E. Sandra Navarro T.

enavarrt@uc.cl 


\begin{abstract}
Introduction: Intensive care units are known as high-stress environments for family members, this can be higher when the patient is a son or daughter and the parents must face the disease along with everything that the hospital environment implies. The Parental Stressor Scale Infant Hospitalization (PSSIH) instrument is a tool used to measure stressors in Pediatric Intensive Care Units (PICU), however, the scale is not validated in Chile. The objective of this study is to culturally validate and adapt the instrument "the modified Parental Stressor Scale Infant Hospitalization" in mothers/fathers of hospitalized children in the PICU of a University Hospital. Method: Instrumental validation study. After translating and counter-translating the English version of the instrument, a group of 10 expert professionals evaluated the Spanish adaptation. Then, 10 parents of hospitalized children in the PICU evaluated the understanding of the instrument. The psychometric properties of the instrument were evaluated using exploratory factorial analysis and Cronbach's alpha. Results: The Chilean version of the "modified PSSIH" was applied to a sample of 221 parents, with minimal semantic modifications and the expert judges considered the instrument adequate, therefore, it was not necessary to delete any item. The 3 -dimensional solution was chosen, which explained $48.89 \%$ of the total instrument variance. The Cronbach's alpha was $0.885,0.902$, and 0.703 respectively for each dimension. Conclusion: The modified PSSIH has proved to be a reliable and valid instrument in a sample of Chilean children hospitalized in a Pediatric Intensive Care Unit of a university hospital. The name in Spanish of the scale is "Escala de Evaluación de Estresores Parentales en niños hospitalizados en Unidades de Cuidados Intensivos Pediátricos” (EEEP - UCIP).
\end{abstract}

\section{Keywords:}

Parental stress;

hospitalization;

pediatric intensive care;

parental stressors

\section{Introducción}

El ingreso de un hijo/a a una Unidad de Cuidados Intensivos Pediátricos (UCIP) es una situación que genera ansiedad y estrés en los padres, alterando la ejecución del rol parental en los momentos donde más lo requiere su hijo/a. Comprender e identificar cuáles son los factores que pueden facilitar el establecimiento y mantención de una intervención terapéutica del equipo de salud es un imperativo ético en el establecimiento de cuidados humanizados en UCIP ${ }^{1}$.

Hay muchas causas generadoras de estrés en un niño hospitalizado, en sus padres y su familia que pueden evitarse como son: el desconocimiento acerca de las implicancias de la enfermedad, de los procedimientos médicos o de las normas y rutinas en el contexto hospitalario. Actualmente en Chile aún existe en las UCIP restricción de tiempo para los padres o cuidadores para acompañar al niño durante las $24 \mathrm{~h}$ del día ${ }^{2}$.

Un estudio realizado por Ramirez, Navarro, Clavería, Molina y $\operatorname{Cox}^{3}$ en 217 padres/madres de niños ingresados en una UCIP de la Región Metropolitana, identificaron los principales estresores parentales que tenía esa Unidad, los que fueron agrupados en tres dimensiones: Clínica, Emocional y Comunicación con el equipo profesional, siendo la dimensión clínica el mayor estresor. Estos resultados corresponden al análisis secundario del proyecto de Investigación Docente Asistencial \# 201403 de Escuela de Enfermería UC durante el proceso de "Validación del instrumento "The Parental Stressor Scale Infant Hospitalization al español" (PSSIH modificado).
Este instrumento, fue el único encontrado al realizar la búsqueda en bases de datos científicas, los resultados en su mayoría fueron instrumentos que evalúan estrés en los padres y/o madres, pero no miden estresores parentales, se encontró solo un trabajo que lo mide en la situación de hospitalización de un hijo, desarrollado por Carter y Miles ${ }^{4}$, denominado "The Parental Stressor Scale Infant Hospitalization" (PSSIH) conformado por 28 items, agrupados en 6 factores: a) aspecto del niño; b)luces y sonidos; c)procedimientos; d)comunicación con el staff profesional; e)conducta y respuesta emocional del niño y f) rol parental. Esta escala fue modificada por Saied ${ }^{5}$ en su tesis doctoral "Stress, Coping. Support and Adjustment among Families of CHD Children in PICU After Heart Surgery" quien incorpora el factor "Conducta de los profesionales" que permite conocer y evaluar la relación del personal clínico de salud con los padres ${ }^{6-11}$, quedando conformado el instrumento por 7 factores y 37 items, instrumento que se utiliza en esta investigación.

El objetivo del presente estudio es validar el instrumento The Parental Stressor Scale Infant Hospitalization modificado por Saied, para la población chilena.

\section{Metodología}

\section{Diseño}

Validación del instrumento de medición "The Parental Stressor Scale Infant Hospitalization" modificado ("PSSIH modificado") para la población chilena. 


\section{Proceso de adaptación y validación del PSSIH modificado}

Se realizaron dos traducciones independientes al español, posteriormente dos contra traducciones por ingleses nativos. Luego analizados individualmente por los miembros del equipo de investigación se consensuaron las opiniones en un instrumento definitivo que se envió a los profesionales expertos.

El proceso de validación una vez traducida la escala fue llevado a cabo en forma secuencial: 1) validez de contenido; 2) adaptación lingüística; 3 ) pre-test de la versión adaptada del instrumento; 4) validez de constructo y análisis de propiedades psicométricas.

\section{Instrumentos y recolección de datos}

El PSSIH modificado consta de 37 ítems agrupados en 7 factores, con una modalidad de respuesta tipo Likert: 1) aspecto del hijo; 2) imágenes o sonidos; 3) procedimientos o intervenciones; 4) conducta del equipo profesional; 5) comunicación del equipo profesional; 6) comportamiento y/o respuesta emocional del hijo; 7) rol de los padres.

\section{Validez de Contenido y adaptación lingüística}

En esta etapa, el PSSIH modificado contra traducido, fue evaluado por 10 profesionales expertos (3 Enfermeras Pediátricas, 2 Pediatras Intensivistas, 2 Psicólogas, 1 médico Anestesista, 1 Neonatólogo y 1 Enfermera Matrona) quienes contestaron un cuestionario respecto de la adecuación del ítem al constructo medido, comprensión y redacción. El análisis de datos consideró la estimación del Coeficiente de Validez de Contenido (CVC) y el Índice de Lynn (IL) para cada ítem, utilizando como criterio de adecuación del ítem un mínimo de 0,6 para el CVC y 0,8 para el IL.

Posteriormente se incorporan los cambios sugeridos por los expertos en redacción y se agrega el ítem "que una máquina respire por mi hijo" al factor "procedimientos e intervenciones" y una pregunta abierta. Después se efectuaron 10 entrevistas a madres/padres de niños hospitalizados en UCIP y se les pidió que evaluaran la claridad del lenguaje, conceptos, redacción y comprensión de cada pregunta. Con las sugerencias se modificó el instrumento obteniéndose la tercera versión en español, que fue piloteada en 10 madres/ padres de niños hospitalizados en la UCIP. En esta última etapa, el instrumento no tuvo modificaciones, obteniéndose la versión final en español de la Escala de Evaluación de Estresores Parentales en UCIP quedando conformada por 38 ítems más una pregunta abierta.

\section{Estudio de validez y confiabilidad}

El tamaño muestral fue determinado según criterio de Brislin que señala un mínimo de 5 sujetos por cada ítem del instrumento. El estudio se realizó en la UCIP de un hospital Universitario de la Región Metropolitana, el tiempo de reclutamiento fue 7 meses (octubre 2015-abril 2016) hasta completar la muestra requerida.

\section{Validez de constructo}

Se utilizó análisis factorial exploratorio, se analizó los ítems en función de su asimetría presentando 18 de los 38 ítems niveles excesivos de asimetría (coeficiente de asimetría superior a 1 en valor absoluto) por lo que el análisis factorial se realizó sobre una matriz policórica, utilizando el método de extracción de mínimos cuadrados no ponderados. Para decidir la cantidad de dimensiones óptimos, se utilizó el criterio de contraste de caída y el análisis paralelo de Horn, en conjunto con un análisis de la varianza explicada y el estadístico de bondad de ajuste Root Mean Square of Residuals (RMSR), considerando un máximo para este indicador de 0.0674. Para estimar la pertenencia de los ítems a la dimensión se utilizó la rotación oblicua oblimín, pues las dimensiones correlacionaron entre sí ( $\mathrm{r}$ superior a $0,4)$ y se utilizó como criterio para incorporar un ítem a la dimensión una correlación mínima de 0,3 .

\section{Estudio de confiabilidad}

Para evaluar consistencia interna se utilizó Alfa de Cronbach incorporando, además, un análisis de la capacidad discriminativa de los ítems por medio de la correlación de cada uno de los reactivos con el puntaje de cada dimensión del instrumento. Se consideró para su interpretación que valores entre 0,60 y 0,69 representan una confiabilidad aceptable, de 0,70 a 0,79 una confiabilidad alta y superior a 0,8 una confiabilidad óptima.

\section{Análisis de los datos}

Para las variables sociodemográficas se calcularon estadísticos descriptivos utilizando el software estadístic SPSS (SPSS para Windows, versión 22, 2012; SPSS Inc., Chicago, IL, USA), mientras que para el análisis factorial del instrumento, se utilizó el software estadístico FACTOR.

Este proyecto cuenta con la aprobación del Comité de Ética Ciéntífico-Medicina Universidad Católica de Chile y los participantes firmaron un Consentimiento informado.

\section{Resultados}

El estudio incorporó un total de 221 participantes.

\section{Evaluación de la validez de Contenido, adecuación lingüística y pilotaje}

Los jueces evaluaron de manera positiva todos los ítems del instrumento. El CVC mínimo registrado para 
un ítem fue de 0,75 y para el IL fue de 0,85 , concluyendo que ningún ítem fue eliminado en esta etapa, sólo se introdujeron algunos cambios menores en el fraseo. Respecto de la adecuación lingüística se registraron cambios menores en los ítems: 1, 2, 3, 4, 5, 8, 9, 13, $20,23,25,32$. Respecto del pilotaje, el tiempo medio de respuesta del cuestionario fue $25 \mathrm{~min}$, sin referir problemas con la autoaplicación. La tabla 1 muestra el instrumento original y la versión posterior a validación de expertos, adecuación lingüística y pilotaje.

\section{Evaluación de validez de constructo, confiabilidad, discriminación y homogeneidad.}

\section{Caracterización de la muestra:}

\section{Características del niño}

Edad de los niños 0 -18 años, con una media de 2,98 y una desviación estándar 4,3 (tabla 2). Respecto a la hospitalización, el $44 \%$ era la primera vez que estaba hospitalizado, el $34 \%$ había tenido 3 o más y el 61,5\%

Tabla 1. Ítems del instrumento original y después del proceso de validación de expertos, adecuación lingüística y pilotaje

Ítems original
Below is a list of items that might describe your
ance. Using the following rating scale, circle the
express how stressful these things have been for you
1. Puffiness of my child
2. Color changes in my child (Pale, blue or yellow)
3. Child appearing cold

Below is a list of Sights and Sounds in an intensive care unit. Circle the number that best express how stressful each of these items has been for you

1. Seeing the heart on the monitors

2. The sound of monitors and equipment

3. The other sick children in the room

4. The sudden sounds of monitor alarms

Below is a list of Procedures that may have been done to your child. Circle the number that best express how stressful these procedures have been for you

1. Tubes in my child

2. Suctioning

3. Putting needles in my child for fluids, procedures or tests

4. Making my child cough and deep breath/pounding and clapping on my child's chest

5. Injections/ shots

6. Bruises, cuts, incisions on my child

Below is a list of items that relate to how Professional staff (doctors and nurses) may Communicate with you about your child's illness. Please indicate the stress level of these items

1. Explaining things too fast

2. Using words I don't understand

3. Tellingme different (conflicting) things about my child's condition

4. Not telling me what is definitely wrong with my child

5. Not talking to me enough

Below is a list of Behaviors and emotional responses that your child may have exhibited while in the intensive care unit. Using the same scale as above, how stressful were things for you?
Ítems modificado

A continuación encontrará una lista de ítems que podrían describir el aspecto de su hijo. Usando la siguiente escala de medición, encierre en un círculo el número que mejor representa lo estresante que estas situaciones han sido para usted

1. Observar el cuerpo hinchado de mi hijo

2. Cambios de color en la piel de mi hijo (pálido, azul o amarillo) y/o presencia de moretones

3. Parecía que mi hijo tenía frío

A continuación, encontrará una lista de ítems que muestran situaciones que pudo haber observado durante la hospitalización de su hijo en la UCIP, tales como imágenes o sonidos. Indique que tan estresantes fueron cada uno de los factores para usted

1. Ver el funcionamiento de su cuerpo u órganos en los monitores

2. El sonido de los monitores y equipos

3. La presencia de otros niños enfermos en la unidad

4. Los sonidos repentinos de las alarmas de los monitores

A continuación encontrará una lista de ítems que describen una serie de procedimientos o intervenciones que pueden haber sido realizados a su hijo. Indique que tan estresantes fueron para usted

1. Presencia de tubos y sondas en mi hijo

2. Aspiración de secreciones u otros líquidos

3. Uso de agujas en mi hijo para administrar sueros, transfusiones, procedimientos o exámenes

4. Hacer que mi hijo tosa, respire fuerte y profundamente o realizar compresiones en el pecho de mi hijo

5. Que una máquina respire por mi hijo (ítem nuevo)

6. Cortes o herida operatoria en mi hijo

7. Inyecciones/vacunas

A continuación encontrará una lista de ítems que describen diferentes situaciones de cómo el Equipo Profesional (médicos y enfermeras/os) se comunicó con usted acerca de la enfermedad de su hijo. Indique que tan estresantes fueron para usted

1. Explicaban muy rápido

2. Usaban palabras que no comprendía

3. Me decían versiones distintas (o contradictorias) sobre la condición de mi hijo

4. No entregaban una versión definitiva de lo que le ocurría a mi hijo 5. No hablaban lo suficiente conmigo

A continuación, encontrará una lista de ítems que describen diferentes formas de Comportamiento y/o respuestas emocionales que su hijo puede haber mostrado durante su hospitalización en la UCIP. Indique que tan estresantes fueron para usted 
Tabla 1. Ítems del instrumento original y después del proceso de validación de expertos, adecuación lingüística y pilotaje (continuación)

Ítems original
1. Confusion
2. Rebellious or uncooperative behavior
3. Crying or whining
4. Demanding
5. Acting or looking as if in pain
6. Restlessness
7. Inability to talk or cry
8. Fright
9. Anger
10. Sadnessor depression

Below is a list of Behaviors of the professional staff (doctors and nurses) that you may have observed. Circle the number that best express how stressful these items have been for you

1. Joking, laughing, or talking loudly

2. Not talking to me enough

3. Toomany different people(doctors, nurses, staff) talking to me

4. Not telling me names or who they are

These items related to Parental Roles. How stressful have the following been for you?

1. Not taking care of my child my self

2. Not being able to visit my child when I wanted

3. Not being able to be with my crying child

4. Not being able to hold my child

Using the same rating scale, how stressful, in general, has the total intensive care unit experience been for you?

Ítems modificado
1. Confuso o desorientado.
2. Comportamiento rebelde o de poca cooperación.
3. Llanto y quejidos.
4. Demandante (muy requirente de atención).
5. Mostrando o evidenciando dolor.
6. Inquietud o Intranquilidad.
7. Incapacidad para hablar o llorar.
8. Miedo.
9. Rabia.
10. Tristeza o depresión.

A continuación, encontrará una lista de ítems que describen una serie de Conductas del Equipo Profesional (médicos y enfermeras/ os) que usted puede haber observado durante la hospitalización de su hijo en la UCIP

1. Estar bromeando, riendo o hablando fuerte.

2. No conversaban lo suficiente conmigo.

3. Distintas personas (médicos, enfermeras/os, personal) hablando conmigo.

4. No indicarme sus nombres o quienes eran al atender a mi hijo.

Los siguientes ítems se relacionan al rol de los padres. Indique qué tan estresantes fueron los siguientes factores para usted

1. No poder cuidar a mi hijo yo mismo

2. No poder visitar a mi hijo cuando yo quiero

3. No poder estar cuando mi hijo está llorando

4. No poder sostener o tomar en brazos a mi hijo

Utilizando la misma escala de evaluación, ¿Qué tan estresante, en general, ha sido la experiencia para usted en la Unidad de Cuidados Intensivos Pediátricos?

¿Existió alguna otra situación que usted considero estresante y que no fue mencionada en este cuestionario?, por favor descríbala: (ítem nuevo) tenía un ingreso programado. En relación a las causas, el $60 \%$ corresponde a problemas cardiacos, el $14,2 \%$ problemas neurológicos y el resto corresponde a oncológico, respiratorio y otra.

\section{Características del cuidador}

Edad entre 18 y 61 años, con una media de 34,6. El $61 \%$ son madres, nivel educacional universitario $62,7 \%$. Respecto del estado civil, casadas $53,4 \%$ y el $64,5 \%$ tienen otro hijo además del que está hospitalizado. El 57,6\% vive en la Región Metropolitana (tabla 3).

\section{Análisis factorial}

Se utilizó el método de extracción de factores de mínimos cuadrados en el programa FACTOR para el análisis de matrices policóricas. La adecuación de la matriz policórica estimó un Kaiser Meyer Olkin $(\mathrm{KMO})$ de $0,866>$ a 0,8 que indica que las condiciones para realizar el análisis son óptimas. Además, la prueba
Tabla 2. Características del niño

\begin{tabular}{llcc}
\hline Variable & Categoría & $\mathrm{n}$ & $\%$ \\
\hline Género & Hombre & 115 & 53,2 \\
& Mujer & 101 & 46,8 \\
& Total válido & 216 & 100 \\
Cantidad de & 1 & 96 & 44 \\
hospitalizaciones & 2 & 48 & 22 \\
& 3 o más & 74 & 34 \\
Ingreso & Total válido & 218 & 100 \\
& Programado & 134 & 61,5 \\
& Urgencia & 84 & 38,5 \\
Área & Total válido & 218 & 100 \\
& Cardiología & 115 & 68 \\
& Neurología & 24 & 14,2 \\
& Oncológico & 10 & 5,9 \\
& Respiratorio & 11 & 6,5 \\
& Otra & 9 & 5,4 \\
& Total válido & 169 & 100 \\
\hline
\end{tabular}


Tabla 3. Características del cuidador

\begin{tabular}{|c|c|c|c|}
\hline Variable & Categoría & $n$ & $\%$ \\
\hline \multirow[t]{3}{*}{ Parentesco } & Madre & 133 & 61 \\
\hline & Padre & 85 & 39 \\
\hline & Total válido & 218 & 100 \\
\hline \multirow[t]{6}{*}{ Escolaridad } & Básica & 7 & 3,2 \\
\hline & Media & 51 & 23,5 \\
\hline & Técnica & 54 & 24,9 \\
\hline & Universitaria & 82 & 37,8 \\
\hline & Postgrado & 23 & 10,6 \\
\hline & Total válido & 217 & 100 \\
\hline \multirow[t]{4}{*}{ Estado civil } & Soltero & 88 & 40,6 \\
\hline & Casado & 116 & 53,4 \\
\hline & Separado & 13 & 6 \\
\hline & Total válido & 217 & 100 \\
\hline \multirow[t]{4}{*}{ Número de hijos } & 1 & 77 & 35,5 \\
\hline & 2 & 71 & 32,7 \\
\hline & 3 o más & 69 & 31,8 \\
\hline & Total válido & 217 & 100 \\
\hline \multirow[t]{7}{*}{ Actividad laboral } & Independiente & 42 & 19,4 \\
\hline & Dependiente & 88 & 40,6 \\
\hline & Dueña de casa & 38 & 17,5 \\
\hline & Cesante & 4 & 1,8 \\
\hline & Licencia médica & 40 & 18,4 \\
\hline & Otro & 5 & 2,3 \\
\hline & Total válido & 217 & 100 \\
\hline \multirow[t]{3}{*}{ Zona } & Urbano & 204 & 94 \\
\hline & Rural & 13 & 6 \\
\hline & Total válido & 217 & 100 \\
\hline \multirow[t]{3}{*}{ Región } & Metropolitana & 125 & 57,6 \\
\hline & Otra & 92 & 42,4 \\
\hline & Total válido & 217 & 100 \\
\hline
\end{tabular}

de Bartlett dio un valor 4.498,4 asociada a un $\mathrm{p}<0,001$ es decir, la matriz policórica a analizar no es una matriz identidad, porque los ítems si correlacionan entre sí.

Respecto a la cantidad de factores, el análisis paralelo de Horn mostró que 3 factores presentan un auto valor superior al proporcionado por el azar. Estos factores explican el $48,894 \%$ de la varianza total del instrumento y es congruente con el criterio de contraste de caída como se puede observar en el gráfico de sedimentación presentado (gráfico 1). El RMSR para esta solución fue de 0,0627 , menor al criterio de 0,0674 lo que indica un ajuste adecuado de la estructura factorial de 3 dimensiones resultantes.

Respecto de las cargas factoriales, utilizando la rotación oblicua oblimin, el resultado del análisis se presenta en la tabla 4.

En relación a la discriminación y homogeneidad, la observación del comportamiento de los ítems muestra que todos presentan niveles adecuados de discriminación (correlaciones sobre 0,25 en su dimensión), sin embargo, se observa una carga factorial ambigua de la escala original en los ítem: "Incapacidad para hablar o llorar" de la dimensión "Respuestas emocionales del hijo"; "No poder cuidar a mi hijo yo mismo" y "No poder estar cuando mi hijo está llorando" de la dimensión "Rol de padres" que podrían afectar la homogeneidad del instrumento.

Acerca de los factores propuestos por los autores, en comparación con la propuesta en este trabajo, se puede observar que los factores "aspecto del hijo", "imágenes o sonido", "procedimientos o intervenciones" y "conducta del equipo profesional" tienden a agruparse en una sola dimensión, que será llamada

\section{GRÁFICO DE SEDIMENTACIÓN}

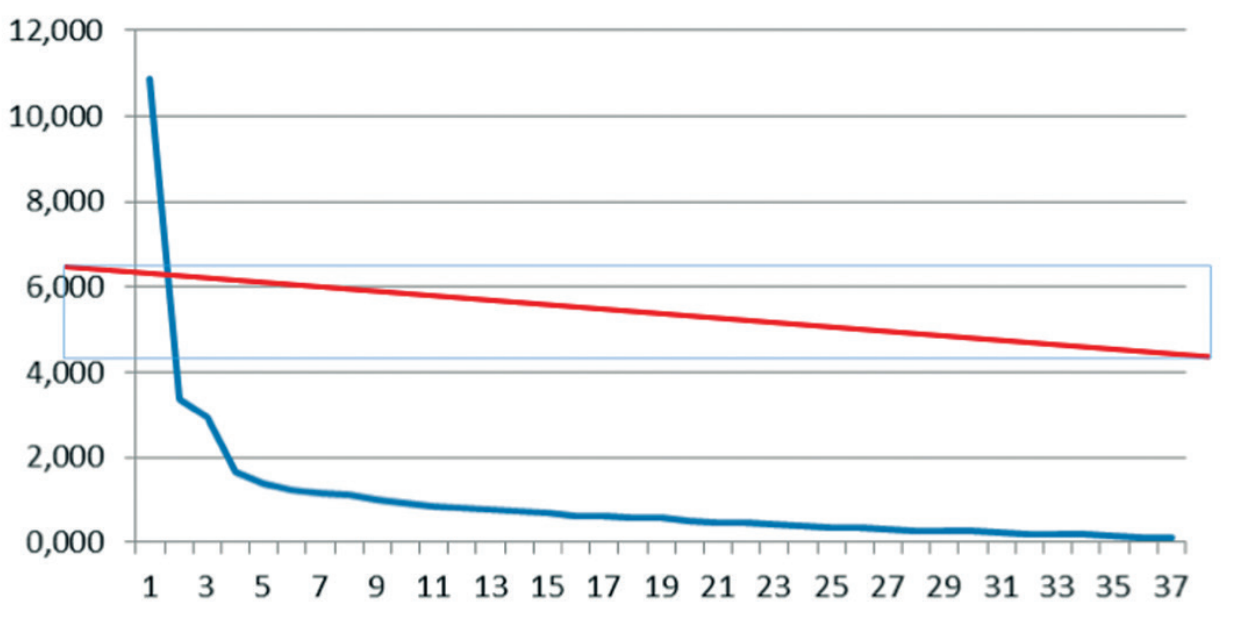


Tabla 4. Cagas factoriales con rotación oblimín (se presentan sólo las cargas factoriales superiores a 0,3)

\begin{tabular}{|c|c|c|c|c|c|c|c|}
\hline & \multicolumn{3}{|c|}{ Dimensión } & & \multicolumn{3}{|c|}{ Dimensión } \\
\hline & 1 & 2 & 3 & & 1 & 2 & 3 \\
\hline ITEM1F1 & & 0,567 & & ITEM1F5 & 0,375 & & \\
\hline ITEM2F1 & & 0,484 & & ITEM2F5 & 0,625 & & \\
\hline ITEM3F1 & & 0,578 & & ITEM3F5 & 0,617 & & \\
\hline ITEM1F2 & & 0,567 & & ITEM4F5 & 0,555 & & \\
\hline ITEM2F2 & & 0,544 & & ITEM5F5 & 0,649 & & \\
\hline ITEM3F2 & & 0,627 & & ITEM6F5 & 0,654 & & \\
\hline ITEM4F2 & & 0,324 & & ITEM7F5 & 0,379 & 0,356 & \\
\hline ITEM1F3 & & 0,692 & & ITEM8F5 & 0,872 & & \\
\hline ITEM2F3 & & 0,513 & & ITEM9F5 & 0,842 & & \\
\hline ITEM3F3 & & 0,511 & & ITEM10F5 & 0,822 & & \\
\hline ITEM4F3 & & 0,423 & & ITEM1F6 & & 0,456 & \\
\hline ITEM5F3 & & 0,422 & & ITEM2F6 & & 0,828 & \\
\hline ITEM6F3 & & 0,712 & & ITEM3F6 & & 0,374 & \\
\hline ITEM7F3 & & 0,763 & & ITEM4F6 & & 0,675 & \\
\hline ITEM1F4 & & & 0,652 & ITEM1F7 & 0,441 & 0,440 & \\
\hline ITEM2F4 & & & 0,583 & ITEM2F7 & & 0,550 & \\
\hline ITEM3F4 & & & 0,682 & ITEM3F7 & 0,366 & 0,365 & \\
\hline ITEM4F4 & & & 0,677 & ITEM4F7 & 0,585 & & \\
\hline ITEM5F4 & & & 0,830 & & & & \\
\hline
\end{tabular}

F corresponde a los factores del instrumento original: F1: Child'Appearance; F2: Sights and sounds; F3: Procedures; F4: Professional staff; F5: Behaviors and emotional; F6: Behaviors of the profesional staff; F7: Parental roles. 1: Dimensión Emocional; 2: Dimensión Clínica; 3: Dimensión Comunicación con el equipo clínico.

Tabla 5. Alfa de Cronbach para las dimensiones originales y para la estructura factorial resultante del presente análisis

\begin{tabular}{lccc}
\hline Dimensión escala original & Alfa de Cronbach & Dimensión resultante & Alfa de Cronbach \\
\hline Aspectos del hijo & 0,713 & Dimensión clínica & 0,885 \\
Imágenes o sonido & 0,767 & & \\
Procedimientos o intervenciones & 0,848 & & \\
Conducta del equipo profesional & 0,703 & Dimensión emocional & 0,892 \\
Respuestas emocionales del hijo & 0,892 & Dimensión comunicación & 0,703 \\
Comunicación equipo de trabajo & 0,703 & Dimensión clínica & 0,904 \\
Rol de padres & 0,819 & Dimensión emocional & 0,902 \\
& & & 0,932 \\
Escala total & & & \\
\hline
\end{tabular}

"Dimensión clínica". El factor original "comportamiento o respuestas emocionales del hijo" y "rol de padres" cargan en la, "Dimensión emocional", respecto del factor rol de padres, esta decisión fue tomada con criterios clínicos ya que los ítems de dicho factor cargan también en la dimensión Clínica. El factor original "comunicación equipo profesional" carga en la dimensión Comunicación equipo profesional, conservando el mismo nombre. Para evaluar la confiabilidad en la dimensión de consistencia interna se utilizó alfa de Cronbach. Se muestra este indicador en la tabla 5 para los factores originales y las dimensiones resultantes del presente análisis.

Así mismo, se aprecia que los análisis de confiabilidad de los factores del instrumento original presentan un nivel adecuado de consistencia interna sobre 
0,7. Analizando las 3 dimensiones resultantes Clínica, Emocional y Comunicación, se evidencia un claro aumento del alfa de Cronbach en los dos primeros factores mencionados con un alfa de 0,885 y de 0,902 respectivamente, en tanto que la tercera dimensión por mantenerse intacto presenta el mismo nivel de consistencia interna que en la estructura factorial original $(0,703)$.

\section{Discusión}

En términos generales, los resultados de esta investigación proporcionan un análisis en relación a la validez del instrumento PSSIH modificado para una muestra de niños chilenos atendidos en una UCIP de un hospital Universitario de la Región Metropolitana. Las adaptaciones realizadas cumplieron los estándares sugeridos por la literatura especializada, considerando diferencias lingüísticas y culturales del contexto. Se logra obtener un instrumento conformado por 38 ítems, agrupados en 3 dimensiones: 1) Clínica compuesta por 4 factores originales (aspecto de su hijo; imágenes y sonidos; procedimientos o intervenciones y conductas del equipo); 2) Comunicación con el equipo clínico, que incluye un factor original (equipo profesional) y 3) Emocional con dos factores originales (comportamiento y/o respuestas emocionales y Rol de los padres). Además, se observaron niveles adecuados de confiabilidad tanto en los factores originales como en las dimensiones resultantes del presente estudio.

Los avances tecnológicos contribuyen eficazmente al tratamiento de los niños en UCIP, sin embargo, hay una relación profesional-paciente cada vez más automatizada, con restricción de acceso a la familia, con foco en los procedimientos y tratamiento medi$\mathrm{co}^{12}$. Por tanto, la evaluación del entorno es esencial constituyéndose en estrategia de cuidado humanizado. La literatura ${ }^{13}$ refiere que implementar actividades que favorezcan la relación de los profesionales con la familia y los niños, permite establecer espacios donde la eficacia técnica se une a la calidad de la atención. Desde esta perspectiva, validar un instrumento que mida estresores parentales responde a la necesidad de las instituciones de salud.

Las dimensiones que aborda este instrumento concuerdan con lo referido en la literatura como estresores ambientales ${ }^{14}$. Involucrar a la familia en los cuidados del hijo gravemente enfermo puede optimizar los resultados del niño, familia y la Institución ${ }^{15}$. Contar con un instrumento validado, permite al equipo de salud evaluar su capacidad de comunicación efectiva, identificar lo que los padres necesitan para involucrarse en el proceso de toma de decisiones y participar en el cuidado de sus hijos.
Así mismo, es una herramienta de apoyo para el desarrollo de indicadores de evaluación de la calidad de la atención, también ayuda a identificar y compartir buenas prácticas.

Respecto a la validación, se encontraron dos estudios en los que se evalúa la estructura factorial del instrumento, una validación al idioma portugués ${ }^{16}$ y otra realizada en una muestra de cuidadores en Estados Unidos ${ }^{17}$.

En el caso de la validación al portugués ${ }^{16}$, sólo incorporaron 26 ítems de los 37 al análisis factorial, el método de extracción utilizado fue el de componentes principales, método criticado por no ser considerado un análisis factorial propiamente tal. Sin embargo, la estructura factorial presentada es congruente con la resultante en este trabajo. Respecto de los resultados del estudio de Agazio y Buckley ${ }^{17}$, también se realizó un análisis de componentes principales, probando soluciones con $5,6,7$ y 8 factores y se decidió por la estructura de 7 factores por calzar adecuadamente con la estructura original, sin embargo, no se reporta la varianza explicada ni otros criterios para delimitar la cantidad de factores, por lo que la comparación de resultados se hace difícil.

Finalmente, en relación a los análisis de consistencia interna, se observó que en general, la escala original presenta valores adecuados de confiabilidad ${ }^{18,19}$ que son congruentes con los encontrados en el presente estudio.

\section{Limitaciones del estudio}

Aplicación del instrumento en una sola UCIP y el muestreo no fue aleatorio.

\section{Conclusión}

Este estudio no solo es un aporte a la investigación en UCIP, sino también, el uso de los instrumentos validados y confiables constituye una herramienta para los equipos de salud que les permite entregar una atención segura y de calidad a los niños y sus padres. Es así como el instrumento The Parental Stressor Scale Infant Hospitalization modificado, ha demostrado ser confiable y válido en una muestra de niños chilenos hospitalizados en una Unidad de Cuidados Intensivos Pediátricos de un hospital universitario, cuyo nombre en español es Escala de Evaluación Estresores Parentales de niños hospitalizados en Unidades Cuidados Intensivos Pediátricos (EEEP-UCIP), constituido por 38 ítems, agrupados en 3 dimensiones: "Dimensión clínica", "Dimensión comunicación con el equipo clínico" y "Dimensión emocional”, más una pregunta abierta. 


\section{Responsabilidades Éticas}

Protección de personas y animales: Los autores declaran que los procedimientos seguidos se conformaron a las normas éticas del comité de experimentación humana responsable y de acuerdo con la Asociación Médica Mundial y la Declaración de Helsinki.

Confidencialidad de los datos: Los autores declaran que han seguido los protocolos de su centro de trabajo sobre la publicación de datos de pacientes.
Derecho a la privacidad y consentimiento informado: Los autores han obtenido el consentimiento informado de los pacientes y/o sujetos referidos en el artículo. Este documento obra en poder del autor de correspondencia.

\section{Conflicto de intereses}

Los autores declaran no tener conflicto de intereses.

\section{Instrumento}

Escala de Evaluación de Estresores Parentales en Unidades de Cuidados Intensivos Pediátricos (UCIP)

(The Parental Stressor Scale: Infant Hospitalization (PSS:IH) Miles \& col., modificado por Saied, validado para población chilena, Proyecto IDA N¹5096. Escuela de Enfermería. Facultad de Medicina Universidad Católica de Chile. Navarro-Tapia, S., Ramírez, M., Clavería, C., Molina, Y.).

Es de gran interés para las enfermeras y otros profesionales que trabajan en la UCIP conocer el efecto que tiene el ambiente de la UCIP en su experiencia como padres.

En el cuestionario que viene a continuación, se incluyen una serie de situaciones que pueden ser consideradas como estresantes para los padres durante la permanencia de su hijo en la UCIP.

Estamos muy interesados en conocer su percepción o experiencia respecto a las situaciones de estrés vividas por usted, en la presente hospitalización de su hijo.

Entendemos por situaciones de estrés, a todas aquellas experiencias vividas que nos hacen sentir ansioso, molesto o tenso. En el siguiente cuestionario, se le pide que marque con un círculo alrededor del número que mejor represente, qué tan estresante ha sido esta situación para usted.

En aquellos ítems que se describen a continuación y que no hayan sido parte de su experiencia, usted debe marcar "No se experimentó" (0).

$$
\begin{array}{ll}
0=\text { No se experimentó } & 3=\text { Moderadamente estresante } \\
1=\text { No fue estresante } & 4=\text { Muy estresante } \\
2=\text { Mínimamente estresante } & 5=\text { Extremadamente estresante }
\end{array}
$$

\begin{tabular}{|c|c|c|c|c|c|c|}
\hline Ítems & $\begin{array}{c}\text { No se } \\
\text { experimentó }\end{array}$ & $\begin{array}{l}\text { No fue } \\
\text { estresante }\end{array}$ & $\begin{array}{l}\text { Mínimamente } \\
\text { estresante }\end{array}$ & $\begin{array}{c}\text { Moderadamente } \\
\text { estresante }\end{array}$ & $\begin{array}{c}\text { Muy } \\
\text { estresante }\end{array}$ & $\begin{array}{c}\text { Extremadamente } \\
\text { estresante }\end{array}$ \\
\hline 1. Observar el cuerpo hinchado de mi hijo & & & & & & \\
\hline $\begin{array}{l}\text { 2. Cambios de color en la piel de mi hijo } \\
\text { (pálido, azul o amarillo) y/o presencia de } \\
\text { moretones }\end{array}$ & & & & & & \\
\hline 3. Parecía que mi hijo tenía frío & & & & & & \\
\hline
\end{tabular}

\section{DIMENSIÓN CLÍNICA}

I. A continuación, encontrará una lista de ítems que podrían describir el aspecto de su hijo. Usando la siguiente escala de medición, encierre en un círculo el número que mejor representa lo estresante que estas situaciones han sido para usted.

\begin{tabular}{|c|c|c|c|c|c|c|}
\hline Ítems & $\begin{array}{l}\text { No se } \\
\text { experimentó }\end{array}$ & $\begin{array}{l}\text { No fue } \\
\text { estresante }\end{array}$ & $\begin{array}{l}\text { Mínimamente } \\
\text { estresante }\end{array}$ & $\begin{array}{l}\text { Moderadamente } \\
\text { estresante }\end{array}$ & $\begin{array}{l}\text { Muy } \\
\text { estresante }\end{array}$ & $\begin{array}{l}\text { Extremadamente } \\
\text { estresante }\end{array}$ \\
\hline $\begin{array}{l}\text { 1. Ver el funcionamiento de su cuerpo u } \\
\text { órganos en los monitores }\end{array}$ & & & & & & \\
\hline 2. El sonido de los monitores y equipos & & & & & & \\
\hline $\begin{array}{l}\text { 3. Los sonidos repentinos de las alarmas de } \\
\text { los monitores }\end{array}$ & & & & & & \\
\hline $\begin{array}{l}\text { 4. La presencia de otros niños enfermos en } \\
\text { la unidad }\end{array}$ & & & & & & \\
\hline
\end{tabular}

II. A continuación, encontrará una lista de ítems que muestran situaciones que pudo haber observado durante la hospitalización de su hijo en la UCIP, tales como imágenes o sonidos. Indique que tan estresantes fueron cada uno de los factores para usted. 
III. A continuación, encontrará una lista de ítems que describen una serie de procedimientos o intervenciones que pueden haber sido realizados a su hijo. Indique que tan estresantes fueron para usted.

\begin{tabular}{|c|c|c|c|c|c|c|}
\hline Ítems & $\begin{array}{c}\text { No se } \\
\text { experimentó }\end{array}$ & $\begin{array}{c}\text { No fue } \\
\text { estresante }\end{array}$ & $\begin{array}{c}\text { Mínimamente } \\
\text { estresante }\end{array}$ & \begin{tabular}{|c} 
Moderadamente \\
estresante
\end{tabular} & $\begin{array}{c}\text { Muy } \\
\text { estresante }\end{array}$ & $\begin{array}{c}\text { Extremadamente } \\
\text { estresante }\end{array}$ \\
\hline \multicolumn{7}{|l|}{ 1. Presencia de tubos y sondas en mi hijo } \\
\hline \multicolumn{7}{|l|}{ 2. Aspiración de secreciones u otros líquidos } \\
\hline \multicolumn{7}{|l|}{$\begin{array}{l}\text { 3. Uso de agujas en mi hijo para adminis- } \\
\text { trar sueros, transfusiones, procedimien- } \\
\text { tos o exámenes }\end{array}$} \\
\hline \multicolumn{7}{|l|}{$\begin{array}{l}\text { 4. Hacer que mi hijo tosa, respire fuerte y } \\
\text { profundamente o realizar compresiones } \\
\text { en el pecho de mi hijo }\end{array}$} \\
\hline \multicolumn{7}{|l|}{ 5. Inyecciones/vacunas } \\
\hline \multicolumn{7}{|l|}{ 6. Cortes o herida operatoria en mi hijo } \\
\hline 7. Que una máquina respire por mi hijo & & & & & & \\
\hline
\end{tabular}

IV. A continuación, encontrará una lista de ítems que describen una serie de Conductas del Equipo Profesional (médicos y enfermeras/os) que usted puede haber observado durante la hospitalización de su hijo en la UCIP.

\begin{tabular}{|c|c|c|c|c|c|c|}
\hline Ítems & $\begin{array}{c}\text { No se } \\
\text { experimentó }\end{array}$ & $\begin{array}{l}\text { No fue } \\
\text { estresante }\end{array}$ & $\begin{array}{l}\text { Mínimamente } \\
\text { estresante }\end{array}$ & $\begin{array}{l}\text { Moderadamente } \\
\text { estresante }\end{array}$ & $\begin{array}{c}\text { Muy } \\
\text { estresante }\end{array}$ & $\begin{array}{l}\text { Extremadamente } \\
\text { estresante }\end{array}$ \\
\hline $\begin{array}{l}\text { 1. Estar bromeando, riendo o hablando } \\
\text { fuerte }\end{array}$ & & & & & & \\
\hline 2. No conversaban lo suficiente conmigo & & & & & & \\
\hline $\begin{array}{l}\text { 3. Distintas personas (médicos, enfermeras/ } \\
\text { os, personal) hablando conmigo }\end{array}$ & & & & & & \\
\hline $\begin{array}{l}\text { 4. No indicarme sus nombres o quienes } \\
\text { eran al atender a mi hijo }\end{array}$ & & & & & & \\
\hline
\end{tabular}

\section{DIMENSIÓN COMUNICACIÓN CON EL EQUIPO CLÍNICO}

V. A continuación, encontrará una lista de ítems que describen diferentes situaciones de cómo el Equipo Profesional (médicos y enfermeras/os) se comunicó con usted acerca de la enfermedad de su hijo. Indique que tan estresantes fueron para usted.

\begin{tabular}{|c|c|c|c|c|c|c|}
\hline Ítems & $\begin{array}{l}\text { No se } \\
\text { experimentó }\end{array}$ & $\begin{array}{l}\text { No fue } \\
\text { estresante }\end{array}$ & $\begin{array}{l}\text { Mínimamente } \\
\text { estresante }\end{array}$ & $\begin{array}{l}\text { Moderadamente } \\
\text { estresante }\end{array}$ & $\begin{array}{c}\text { Muy } \\
\text { estresante }\end{array}$ & $\begin{array}{l}\text { Extremadamente } \\
\text { estresante }\end{array}$ \\
\hline 1. Explicaban muy rápido & & & & & & \\
\hline 2. Usaban palabras que no comprendía & & & & & & \\
\hline $\begin{array}{l}\text { 3. Me decían versiones distintas (o contra- } \\
\text { dictorias) sobre la condición de mi hijo }\end{array}$ & & & & & & \\
\hline $\begin{array}{l}\text { 4. No entregaban una versión definitiva de } \\
\text { lo que le ocurría a mi hijo }\end{array}$ & & & & & & \\
\hline 5. No hablaban lo suficiente conmigo & & & & & & \\
\hline
\end{tabular}

\section{DIMENSIÓN EMOCIONAL}

VI. A continuación, encontrará una lista de ítems que describen diferentes formas de Comportamiento y/o respuestas emocionales que su hijo puede haber mostrado durante su hospitalización en la UCIP. Indique que tan estresantes fueron para usted.

\begin{tabular}{|c|c|c|c|c|c|c|}
\hline Ítems & $\begin{array}{c}\text { No se } \\
\text { experimentó }\end{array}$ & $\begin{array}{l}\text { No fue } \\
\text { estresante }\end{array}$ & $\begin{array}{l}\text { Mínimamente } \\
\text { estresante }\end{array}$ & $\begin{array}{l}\text { Moderadamente } \\
\text { estresante }\end{array}$ & $\begin{array}{c}\text { Muy } \\
\text { estresante }\end{array}$ & $\begin{array}{l}\text { Extremadamente } \\
\text { estresante }\end{array}$ \\
\hline \multicolumn{7}{|l|}{ 1. Confuso o desorientado } \\
\hline \multicolumn{7}{|l|}{$\begin{array}{l}\text { 2. Comportamiento rebelde o de poca } \\
\text { cooperación }\end{array}$} \\
\hline \multicolumn{7}{|l|}{ 3. Llanto y quejidos } \\
\hline $\begin{array}{l}\text { 4. Demandante (muy requirente de aten- } \\
\text { ción) }\end{array}$ & & & & & & \\
\hline
\end{tabular}




\begin{tabular}{|l|l|l|l|l|l|}
\hline 5. Mostrando o evidenciando dolor & & & & & \\
\hline 6. Inquietud o Intranquilidad & & & & & \\
\hline 7. Incapacidad para hablar o llorar & & & & & \\
\hline 8. Miedo & & & & & \\
\hline 9. Rabia & & & & & \\
\hline 10. Tristeza o depresión & & & & & \\
\hline
\end{tabular}

VII. Los siguientes ítems se relacionan al rol de los padres. Indique qué tan estresantes fueron los siguientes factores para usted.

\begin{tabular}{|c|c|c|c|c|c|c|}
\hline Ítems & $\begin{array}{l}\text { No se } \\
\text { experimentó }\end{array}$ & $\begin{array}{l}\text { No fue } \\
\text { estresante }\end{array}$ & $\begin{array}{l}\text { Mínimamente } \\
\text { estresante }\end{array}$ & $\begin{array}{l}\text { Moderadamente } \\
\text { estresante }\end{array}$ & $\begin{array}{c}\text { Muy } \\
\text { estresante }\end{array}$ & $\begin{array}{l}\text { Extremadamente } \\
\text { estresante }\end{array}$ \\
\hline 1. No poder cuidar a mi hijo yo mismo & & & & & & \\
\hline $\begin{array}{l}\text { 2. No poder visitar a mi hijo cuando yo } \\
\text { quiero }\end{array}$ & & & & & & \\
\hline $\begin{array}{l}\text { 3. No poder estar cuando mi hijo está } \\
\text { llorando }\end{array}$ & & & & & & \\
\hline $\begin{array}{l}\text { 4. No poder sostener o tomar en brazos a } \\
\text { mi hijo }\end{array}$ & & & & & & \\
\hline
\end{tabular}

VIII. Utilizando la misma escala de evaluación, ¿Qué tan estresante, en general, ha sido la experiencia para usted en la Unidad de Cuidados Intensivos Pediátricos?

\begin{tabular}{|c|c|c|c|c|c|}
$\begin{array}{c}\text { No se } \\
\text { experimentó }\end{array}$ & $\begin{array}{c}\text { No fue } \\
\text { estresante }\end{array}$ & $\begin{array}{c}\text { Mínimamente } \\
\text { estresante }\end{array}$ & $\begin{array}{c}\text { Moderadamente } \\
\text { estresante }\end{array}$ & $\begin{array}{c}\text { Muy } \\
\text { estresante }\end{array}$ & $\begin{array}{c}\text { Extremadamente } \\
\text { estresante }\end{array}$ \\
\hline & & & & & \\
\hline
\end{tabular}

IX. ¿Existió alguna otra situación que usted consideró estresante y que no fue mencionada en este cuestionario?, por favor descríbala:

\section{Referencias}

1. Thompson V, Hupcey J, Clark M. The Development of Trust in Parents of Hospitalized Children. J Spec Pediatr Nurs. 2003;8(4):137-47.

2. Ochoa B, Polaino-Lorente A. El estrés de los padres como consecuencia de la hospitalización de sus hijos: una revisión. Estudios de Psicología 1999;20:63-64,147161, DOI: $10.1174 / 02109399960256829$.

3. Ramirez, M., Navarro, S., Clavería, C., Molina, Y. \& Cox, A. Estresores parentales en Unidades de Cuidados Intensivos Pediátricos. Rev Chil Pediatr 2018;89(2):182-9.

4. Carter MC, Miles MS, Buford TH, Hassanein RS. Parental environmental stress in pediatric intensive care units. Dimens Crit Care Nurs. 1985;4(3):180-8.

5. Saied H. Stress, Coping, Social Support and Adjustment Among Families of CHD Children in PICU After Heart Surgery. Electronic Thesis or Dissertation. Case Western Reserve University, 2006. Ohio LINK Electronic Theses and Dissertations Center. Mar 2015.

6. Costa JB, Felicetti CR, Costa CR, Miglioranza DC, et al. Fatores estressantes para familiares de pacientes críticamente enfermos de uma unidade de terapia intensiva. J Bras Psiquiatr 2010;59(3):1829.

7. Parra Falcón F, Moncada Z, Oviedo Soto $S$, Marquina M. Estrés en padres de los recién nacidos hospitalizados en la
Unidad de Alto Riesgo Neonatal. Index Enferm 2009;18(1):13-7.

8. Melnyk BM, Alpert-Gillis L, Hensel P, Cable-Beiling R, Rubenstein JS. Helping mothers cope with a critically ill child: a pilot test of the COPE intervention. Res Nurs Health 1997;20(1):3-14.

9. Fernández Castillo A, López Naranjo I. Estrés parental en la hospitalización infantil. Ansiedad estrés 2006;12(1):1-17.

10. Ochoa Linacero B, Polaina Lorente A. El estrés de los padres como consecuencia de la hospitalización de sus hijos: una revisión. Estud Psicol 1999;20(63-64):14762.

11. Ruiz A, Ceriani Cernadas J, Cravedi V, Rodríguez D. Estrés y depresión en madres de prematuros: un programa 
de intervención. Arch Argent Pediatr. 2005;103(1):36-45.

12. Board R, Ryan-Wenger N. Long-term effects of pediatric intensive care unit hospitalization on families with young children. Heart Lung 2002;31(1):53-66.

13. Marques IR, Souza AR. Tecnologia e humanização em ambientes intensivos. Rev Bras Enferm. 2010;63(1):141-4.

14. Shudy M, de Almeida ML, Ly S, Landon C, et al. Impact of pediatric critical illness and injury on families: a systematic literature review. Pediatrics 2006;118
Suppl 3:S203-18.

15. Portella J, Calcagno G, Buss M. Health facility environment as humanization strategy care in the pediatric unit: systematic review. Rev Esc Enferm USP 2014;48(3):527-36 www.ee.usp.br/reeusp/

16. Corlett J, Twycross A. Negotiation of parental roles within family-centred care: A review of the research. J Clin Nurs. 2006;15(10):1308-16. doi:10.1111/j.13652702.2006.01407.x

17. De Souza S, Dupas G, Ferreira Gomes M. Cultural adaptation and validation for the Portuguese language of the Parental Stress Scale:Neonatal Intensive Care Unit (PSS:NICU). Acta Paul Enferm. 2012;25(2):171-6.

18. Agazio J, Buckley K. Revision of a Parental Stress Scale for Use On a Pediatric General Care Unit. Pediatr Nurs. 2012;38(2):82-7.

19. Mansson C, Jakobsson U, Lundqvist P. Translation and psychometric evaluation of a Swedish version of the parental stressor scale PSS: NICU. Scand J Caring Sciences 2016;30:193-201. 